\title{
Unusual Recalcitrant Classic Kaposi's sarcoma in a Non-AIDS Yemeni Man: Is It a Zoonotic!
}

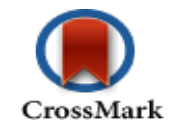

\author{
Al-Kamel Mohamed Ahmed ${ }^{1,2}$ \\ ${ }^{1}$ Regional Leishmaniasis Control Center (RLCC), Yemen \\ ${ }^{2}$ Sana'a University Hospital, Sana'a, Yemen
}

*Corresponding author: Mohamed A. Al-Kamel, MD, Regional Leishmaniasis Control Center, P.O.Box 12692, Sana'a, Yemen. Tel: +967 777223535. E-mail: dralkamel@hotmail.com

\begin{abstract}
I report a case of a 43-year-old non-HIV infected Yemeni shepherd, who presented with a fungating, multipigmented plaque of several, purplish/dark-brownish, friable nodules on the right index finger, cutaneous dissemination, and lymphatic infiltrates.

Chronicity, number and morphology of the lesions, local lymphatic infiltration and the characteristic histopathological findings were identical with Kaposi's sarcoma (KS); the classic variant. Due to rarity of non human immunodeficiency virus (HIV)-associated KS, here I report a case of classic KS in an immune competent middle-aged Asian male. I also raise a question about human KS-associated herpesvirus (KSHV) and its zoonotic potential for cross-species infection.
\end{abstract}

Keywords: Kaposi’s sarcoma; Classic; Herpesvirus; Zoonotic; Yemen
Received date: Feb 27, 2016

Accepted date: June 03, 2016

Published date: June 09, 2016

Citation: Al-Kamel, M.A. Unusual Recalcitrant Classic Kaposi's Sarcoma in aNon-AIDSYemeni Man: Is It a Zoonotic! (2016) Invest Dermatol Venereol Res 2(2): 74- 77.

DOI: $10.15436 / 2381-0858.16 .796$

Abbreviations: KS: Kaposi's Sarcoma; CKS: Classis Kaposi's Sarcoma; HIV: Human Immunodeficiency Virus; AIDS: Acquired Immunodefieciency Syndrome; KSHV: Kaposi's Sarcoma-associated Herpesvirus; HHV-8: Herpesvirus 8

\section{Introduction}

Herpesviridae is a large family of DNA viruses that cause severe diseases in animals, including humans. The members of this family are also known as herpesviruses. There are more than 130 herpesviruses and some are from animals. They are host specific agents that also share many properties that potentially make them capable of crossing the species barriers. In total, there are 8 herpes virus types that infect humans; Kaposi's sarcoma-associated herpesvirus (KSHV), also known as herpesvirus 8 (HHV-8) is a one strongly implicated in the pathogenesis of all types of Kaposi's sarcoma (KS) ${ }^{[1-3]}$.

$\mathrm{KS}$ is a multicentric soft tissue malignancy arising from endothelial cells of blood vessels involving; skin, lymph node, gastrointestinal tract, and lungs. It is endemic in African countries, has higher prevalence in people of Arabian countries and other Mediterranean descent ${ }^{[4-6]}$.

KS is classified into four types; classic (CKS), African endemic, iatrogenic and epidemic (AIDS-related). The course of KS ranges from indolent, with only skin involvement to fulminant with extensive visceral involvement.

The incidence rate of KS among AIDS patients is several thousand-fold higher than among the general population. CKS is not common affecting non-AIDS patients ${ }^{[7]}$.

\section{Case Report}

January $30^{\text {th }}, 2016$, a 43-year-old rural Yemeni man presented with progressively developed livid, fungating, multipigmented plaque (approximately $55 \times 40 \mathrm{~mm}$ ) of several, well-defined, purplish/vinous, friable, tender, nodulo-ulcerative lesions that 
easily bleed on injury, on the palmar aspect of his right index finger (Figure. 1). The condition started 8 months as an erythematous macule that gradually developed to a bigger nodule forming a multifocal tumor. The condition worsen over the time and the lesions progressed to cover most of the finger. Afterwards, asymptomatic violaceous nodule developed under the left eyelid, as well.

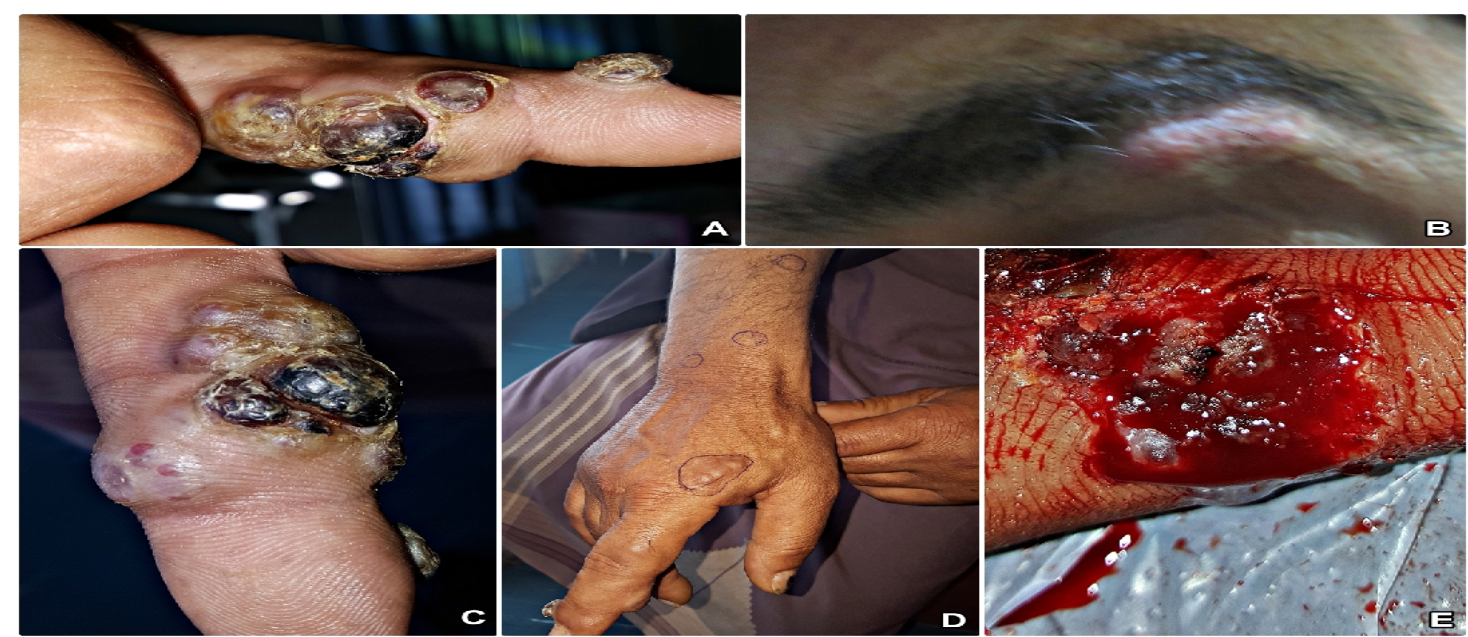

Figure 1: Morphology of lesions on presenting day. (A\&C) Multipigmented fungating plaque and well-defined nodules on the palmaraspect of index finger of right hand. (B) Violaceousnodule under left eyelid. (D) Elevated nodules started to develop along the lymphatic drainage of the affected finger. (E) Profound, fragile, tortuous blood vessels at the base of the biopsy's excised nodule.

Two months ago, numerous, smaller, firm, deep-cutaneous $0.5-1 \mathrm{~cm}$ nodules started to develop along the lymphatic drainage of the same finger, up to the left mid-forearm. No regional lymph nodes, oral or genital involvement.

Direct questioning regarding his occupational history revealed that he is a farmer and a sheep shepherd with animals living in the same household. He reported that some of his sheep have complained of unknown infectious skin lesions, in the form of erosions and blisters that spontaneously rupture spreading the infection to healthy ones.

Medical history revealed that the patient roughly received many courses of antibiotics, corticosteroids and other unknown treatment modalities.

Past history revealed, 16 months before (September $26^{\text {th }}, 2014$ ), the patient presented with similar brownish-red nodules on both aspects of the neighboring right middle finger (but without lymphatic involvement or distal dissemination), of 6 months duration, that was treated and responded well to cryotherapy with complete regression without scarring (Figure. 2). Two weeks later, he noticed similar lesions started to appear on the palmar aspect of the presenting right index finger, and thus the current condition started to develop. History of a previous hand trauma was irrelevant.

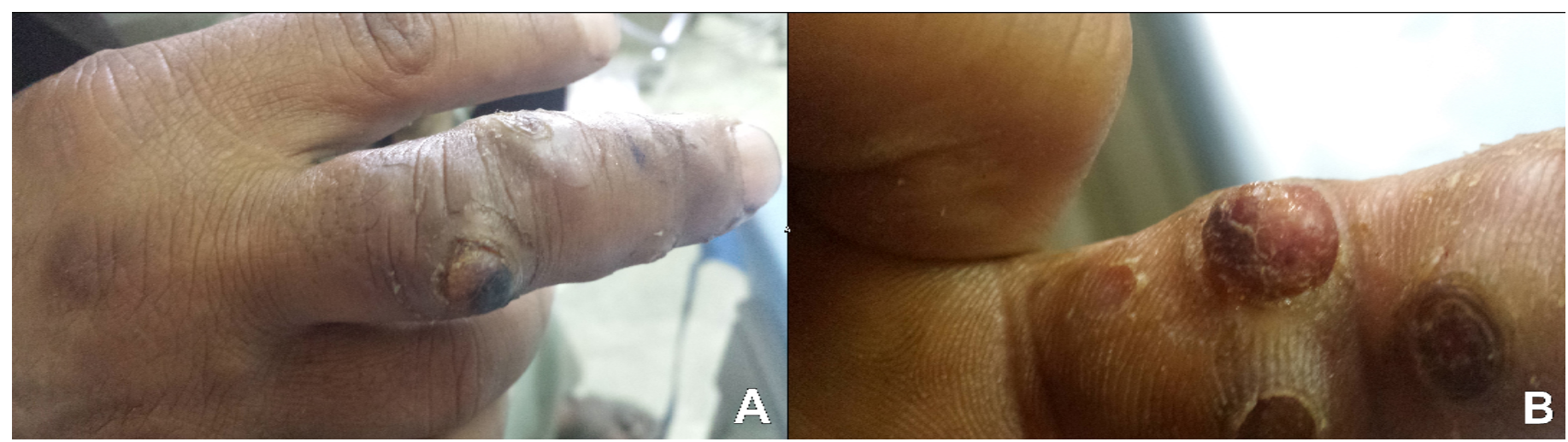

Figure 2:(A\&B): Archived photos showing a previous presentation, 16 months ago, with brownish-red, nodular lesions on both aspects of the neighboring right middle finger. (B) Healing without scar formation after one session of cryotherapy treatment, is observed.

Except for a mild eosinophilia, investigations revealed normal hemogram, HIV, HBV, HCV, LFT, KFT and internal imaging scans.

An excisional $2 \times 1.5 \mathrm{~cm}$ biopsy was obtained. Pending the H\&E stained specimen examination results, differential diagnosis included KS, Orf and Milker's nodules, bacillary angiomatosis, cutaneous lymphoma and leprosy nodule, was taken in consideration.

Histopathological examination (Figure. 3) shows hyperkeratotic epidermis with focal parakeratosis and focal thinning in the middle part. In the dermis, there is a nodule of proliferation of small vascular spaces, some of the rounded, and others slit-like with some small bundles of spindle cells, red blood cells extravasation, is also seen, and hemosiderin and dilated vascular spaces in the periphery are occasionally seen. These findings suggest KS, with uncertain malignant potential. PCR and HHV-8 testing were not carried out. 


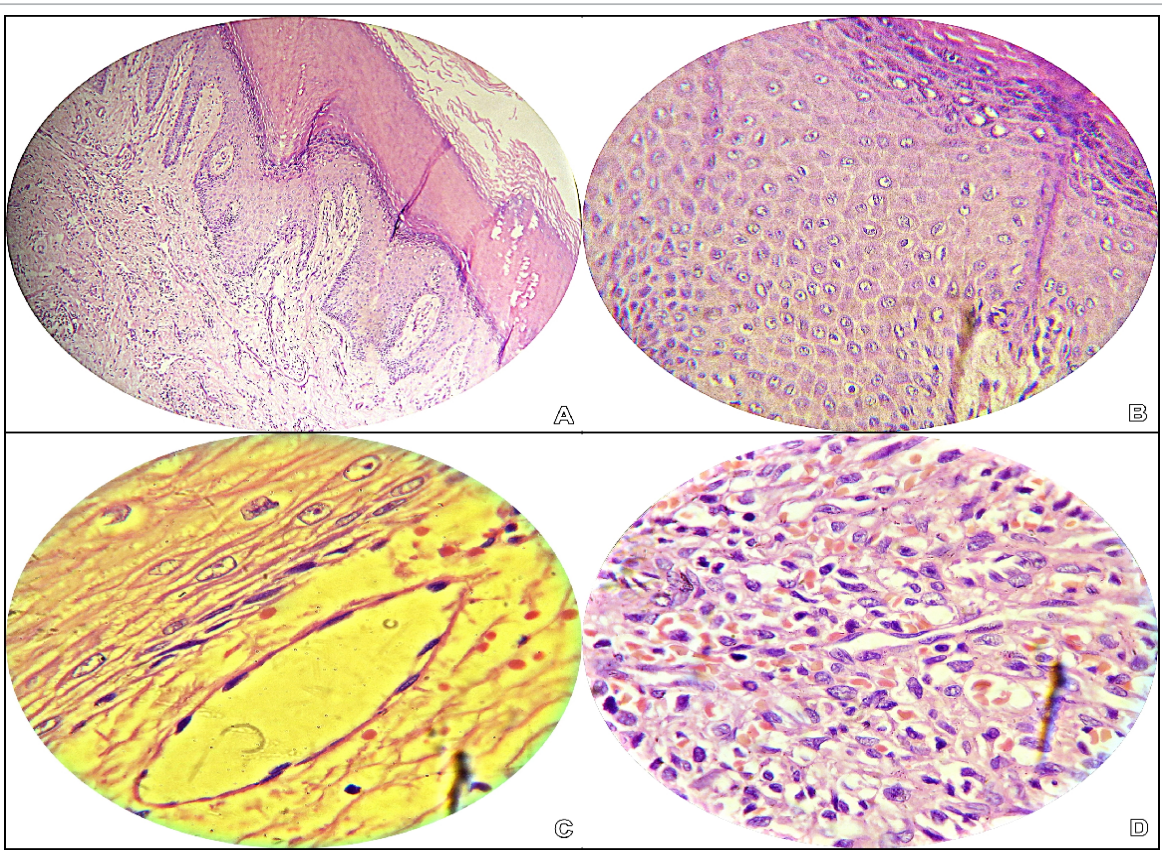

Figure 3: Photomicrographs showing the characteristic histologic features of Kaposi’s sarcoma (H\&E;10,40,100). (A\&B) Hyperkeratotic epidermis with focal parakeratosis and focal thinning in the middle part. (C\&D)In the dermis, there is a nodule of proliferation ofdiffuse small vascular spaces, some of the rounded, and others slit-like with some bundles of spindle cells (invading the dermis), in association with extravasated erythrocytes, hemosiderin and fibrosis.Irregular vascular spaces are seen developing around pre-existing vascular structures.

On basis of the clinical and characteristic histopathological findings, a definite diagnosis of Kaposi's sarcoma was made, and the patient was referred to a general hospital for further management.

\section{Discussion}

Kaposi's sarcoma (KS) is a soft tissue cancer; characterized histologically by endothelial-lined vascular spaces and spindle-shaped cells. It can present with cutaneous lesions with or without internal involvement. The cutaneous lesions can be solitary, localized or disseminated. The erythematous to violaceous cutaneous lesions seen in KS have several morphologies; patch, plaque, nodular, lymphadenopathic, infiltrative, and florid. Typically, the disease is multifocal and recurs despite treatment. The diagnosis can be made with a tissue biopsy and, if clinically indicated, internal imaging should be done $\mathrm{e}^{[3,8]}$.

Endemic KS has been originally described in young African people, mainly from sub-Saharan Africa, however it may be present worldwide. This variant often affects human immunodeficiency virus (HIV)-negative individuals, including children, and can take an aggressive form involving the lymph nodes ${ }^{[9]}$.

Iatrogenic KS is a type associated with immunosuppressive drug therapy, typically seen in renal allograft recipients. This variant tends to be aggressive, involving lymph nodes, mucosa, and visceral organs in about half of patients, sometimes in the absence of skin lesions ${ }^{[7]}$.

Epidemic KS (AIDS-associated) typically presents with cutaneous lesions, with a predilection for the head, neck, back, trunk and mucous membranes. In more advanced cases, they can be found in the stomach and intestines, the lymph nodes, and the lungs ${ }^{[8]}$.

Classic Kaposi sarcoma (CKS) was described in 1872 by Moriz Kaposi, as an idiopathic, multipigmented sarcoma of the skin. Itis a rarevariant, typically presents much more common in men, tends to be indolent and chronic, and though in the majority of cases it is benign.

CKS typically appears initially on the hands and feet as reddish, violaceous, or bluish-black well-demarcated, painless macules and patches that progress slowly and may merge into nodules, plaques or fungi-formed, brownish-red tumors. The latter are characterized mostly by spindle-shaped tumor cells that are compressed with vascular slits, often in association with extravasated erythrocytes, hemosiderin and fibrosis. Unilateral involvement generally is observed at the onset of KS; however, subsequently the disease tends to be bilateral and multifocal with centripetal spreading. As KS lesions progress, they become brownish in color and may display verrucous and hyperkeratotic surfaces. At advanced stages of the disease, large, partly eroded, and ulcerated plaques that involve the feet, hands, and even entire extremities can occur. Enlargement of the lymph nodes, both superficial and deep, also is an early manifestation of the disease and may precede the cutaneous manifestations. A small percentage of patients may have visceral and/or mucosal involvement ${ }^{[6,7,10]}$.

Unlike KS in AIDS patients, non-HIV associated KS is a rather localized process which rarely involves lymph nodes or organs and in most cases responsive on local or systemic therapeutic strategies. African endemic KS, AIDS-related, and iatrogenic $\mathrm{KS}$ tend to be more aggressive than $\mathrm{CKS}^{[7,11]}$.

Sheep and cattle parapox virus infections cause in human beings diseases of very similar aspect, named Orf and milker's 
nodules, respectively. However, these viral conditions are benign and self-limiting(4 to 9 weeks).

Kaposi's sarcoma-associated herpesvirus (KSHV) can be transmitted sexually, during labor and delivery or transplacentally, and by other means. The exact routes of transmission are not known, although KSHV has been detected in both saliva and semen from infected persons ${ }^{[6]}$.

Taken together, herpesviruses are highly species-specific and present an almost negligible zoonotic threat. However, close contact between infected animal hosts and humans may lead to transmission and replication of other Herpesviridae members which increase the risk for a zoonotic infection ${ }^{[1,12]}$.

As with other cell-transforming human DNA viruses, infection with KSHV/HHV8 alone is not sufficient for the development of KS and additional cofactors are required. Many contributory factors undoubtedly have etiologic and pathogenic significance in the development of classic KS. Geographic location, ethnicity, time interval, age, and gender heavily influence disease incidence ${ }^{[10]}$.

Many treatments have been used to treat classic KS, although no definitive cure is known at present. Surgery, formerly recommended, is no longer indicated apart from tissue analysis. The tendency toward multifocality makes radiation therapy or chemotherapy, or both, the preferred mode of treatment. Lesions of KS are highly radiosensitive ${ }^{[2]}$.

KS is a very rare disease in Yemen. The only available epidemiological data indicate the incidence of KS compared to other cancers in women of all ages in Yemen (estimations for 2012) as one per 1000,000 per year; incidence among men haven’t been reported $^{[13]}$.

The diagnosis of KS in the current case was made on the ground of chronicity, number and morphology of the lesions, locallymphatic infiltration and the specific histopathological findings.

Contrary to other reports, the classic KS is usually associated with HIV infection, this report has demonstrated otherwise. The clinical picture of the current disease is strongly related to cutaneous, nodular, classic KS variant, with anaplastic potential. Risk factors for such disease in an immunocompetent patient are unknown. Genetic and ethnic predisposition have been reported ${ }^{[5]}$.

This report raises a question about the relationship between herpesviruses of animals and humans, and their zoonotic potential, considering patient's prolonged contact with infected sheep. Such cross species infections are generally rare, but still unpredictable and possible ${ }^{[1,2]}$.

\section{Conclusion}

This report may open new avenues for the research of KS. To the best of author's knowledge, such a case of KS has not been reported in Yemeni literatures. Awareness of KS clinic pathological features is important to avoid unnecessary interventions.

Conflict of Interest Disclosure: None declared.

\section{References}

1. Kaposi, M. Idiopathisches multiples Pigmentsarkom der Haut. (1872) Arch Dermatol Syph 4(2): 265-272.

2. Wozniakowski, G., Samorek-Salamonowicz, E. Animal herpesviruses and their zoonotic potential for cross-species infection. (2015) Ann Agric Environ Med 22(2): 191-194.

3. Carter, J., Saunders, V. Virology: Principles and Applications. (2016) John Wiley \& Sons; 2nd edition. ISBN 978-0-470-02386-0.

4. Rafi, A. J., Koul, A.P., Ahmed, M., et al. Kaposi Sarcoma in a Non HIV Patient. (2008) Int J Health Sci (Qassim) 2(2): $153-156$.

5. Tan, H.H., Goh, C.L. Viral infections affecting the skin in organ transplant recipients: Epidemiology and current management strategies. (2006) Am J Clin Dermatol 7(1): 13-29.

6. Jalilvand, S., Shoja, Z., Mokhtari-Azad, T., et al. Seroprevalence of Human herpesvirus 8 (HHV-8) and incidence of Kaposi's sarcoma in Iran. (2011) Infect Agent Cancer 6: 5.

7. Iscovich, J., Boffetta, P., Franceschi, S., et al. Classic Kaposi sarcoma: epidemiology and risk factors. (2000) Cancer 88(3): 500-517.

8. Antman, K., Chang, Y. Kaposi's Sarcoma. (2000) N Engl J Med 342: 1027-1038.

9. Cook-Mozaffari, P., Newton, R., Beral, V., et al. "The geographical distribution of Kaposi sarcoma and of lymphomas in Africa before the AIDS epidemic". (1998) Br J Cancer 78(11): 1521-1528.

10. William, J.D., Berger, T., Dirk, M. Andrews' Diseases of the Skin: Clinical Dermatology. (10th ed.). (2006) ISBN 080892351X.

11. Jakob, L., Metzler, G., Chen, K.M., et al. Non-AIDS Associated Kaposi's Sarcoma: Clinical Features and Treatment Outcome. (2011) PLoS One 6(4): e18397.

12. Tischer, B.K., Osterrieder, N. Herpesviruses - a zoonotic threat? (2010) Vet Microbiol 140(3-4): 266.

13. Bruni, L., Barrionuevo-Rosas, L., Albero, G., et al. ICO Information Centre on HPV and Cancer (HPV Information Centre). Human Papillomavirus and Related Diseases in Yemen. (2016) Summary Report.

Online ISSN: $2381-0858$

Journal Title: Investigative Dermatology and Venereology Research Journal Short Name: Invest Dermatol Venereol Res
Ommega Online Publishers

E-mail: editor.dermatology@ommegaonline.org

Website: www.ommegaonline.org 